\title{
Adaptation of Smart Antenna with AODV Routing Protocol for Ad Hoc Wireless Networks
}

\author{
Mebratu Fana Bedasa', Asrat Sime Bedada1, Wesenu Bekele Mulatu² \\ ${ }^{1}$ Department of Computer Science, College of Computing, Madda Walabu University, Bale Robe, Ethiopia \\ ${ }^{2}$ Department of Information System, College of Computing, Madda Walabu University, Bale Robe, Ethiopia \\ Email:mebratu.fana@yahoo.com,asrt789@gmail.com,wesenubekele1@gmail.com
}

How to cite this paper: Bedasa, M.F. Bedada, A.S. and Mulatu, W.B. (2020) Adaptation of Smart Antenna with AODV Routing Protocol for Ad Hoc Wireless Networks. Open Access Library Journal, 7: e6504.

https://doi.org/10.4236/oalib.1106504

Received: June 7, 2020

Accepted: July 25, 2020

Published: July 28, 2020

Copyright $\odot 2020$ by author(s) and Open Access Library Inc.

This work is licensed under the Creative Commons Attribution International License (CC BY 4.0).

http://creativecommons.org/licenses/by/4.0/

\begin{abstract}
In the recent years, Mobile Ad-Hoc Wireless Networks (MANET) have played a key role in communication system. It is a self-configured and infrastructure-less network. It is used in different application areas like disaster recovery operations, hospitals and agricultural sectors. However, there are a lot of problems that limit the performances of this network for significantly achieving its roles in different areas. To overcome these problems, this paper enhances the performances of MANET in wireless communication system by adapting smart antenna in Ad-hoc On-Demand Distance Vector (AODV) routing protocol combining with multiple network interfaces. The improved new version AODV utilizing directional antenna and multiple network interface compared with Omni-directional antenna is evaluated based on simulations, which was done with the Network Simulator version two (NS2). Through this study, we found out that directional antennas integrating with multiple network interface throughput and end to end delays always outperform Omni-directional antennas in both random and grid scenarios for AODV routing protocol. In high communication paths and long communication distances also directional antennas have significant improvements in contrast to traditional Omni-directional antennas. Finally, we recommend that for increasing throughput and decreasing end to end delay utilizing directional antennas and multiple network interfaces in MANET technology improve the performances of the wireless communication system.
\end{abstract}

\section{Subject Areas}

Applications of Communication Systems, Network Modeling and Simulation

\section{Keywords}

Routing Protocol, MANET, Smart Antenna, Multiple Network Interface 


\section{Introduction}

\section{Background}

MANETs are a recent year wireless technologies research area which achieves better costs, no need for intermediate devices, self-organized, self-manage, and simple to deploy in mobile communication system [1] [2]. Each node in MANET acts as both a host and a router and send packets between nodes that cannot directly communicate with one other. The frequency spectrum of the MANET node has much smaller than a node in a fixed infrastructure network. A MANET has an independent collection of mobile users that communicate over relatively bandwidth-constrained wireless links (Figure 1). The MANET nodes are mobile, the network topology may change rapidly and unpredictably over time. The network is distributed, where all network activity including discovering the topology and delivering messages executed by the nodes themselves and routing functionality incorporated into mobile nodes [1] [2] [3].

This network is very important in a place where no infrastructure is accessible. Today it plays a great role in different areas like defense system, health center, disaster recovery operations, agricultural organization, vehicular networks, ubiquitous computing, sensor networks and others. In wireless network, the process of communication can be dynamically set-up anywhere and anytime without using any pre-existing network infrastructure. In conventional MANETs, to send or receive packets it have been known to use the Omni-directional antenna which leads to lower power efficiencies because of interference caused by the transmission of packets in the unwanted direction and finally this problem deteriorates the entire network performances. Not only this, MANETs have several challenges. The most known MANET challenges are dynamic topology, limited security, limited bandwidth, routing and battery life of the nodes [1] [2]. To solve different problems of MANET, many researchers were found that various algorithms that reduce these problems on different Medium Access Control (MAC) layer, reactive and proactive routing protocols and antenna types.

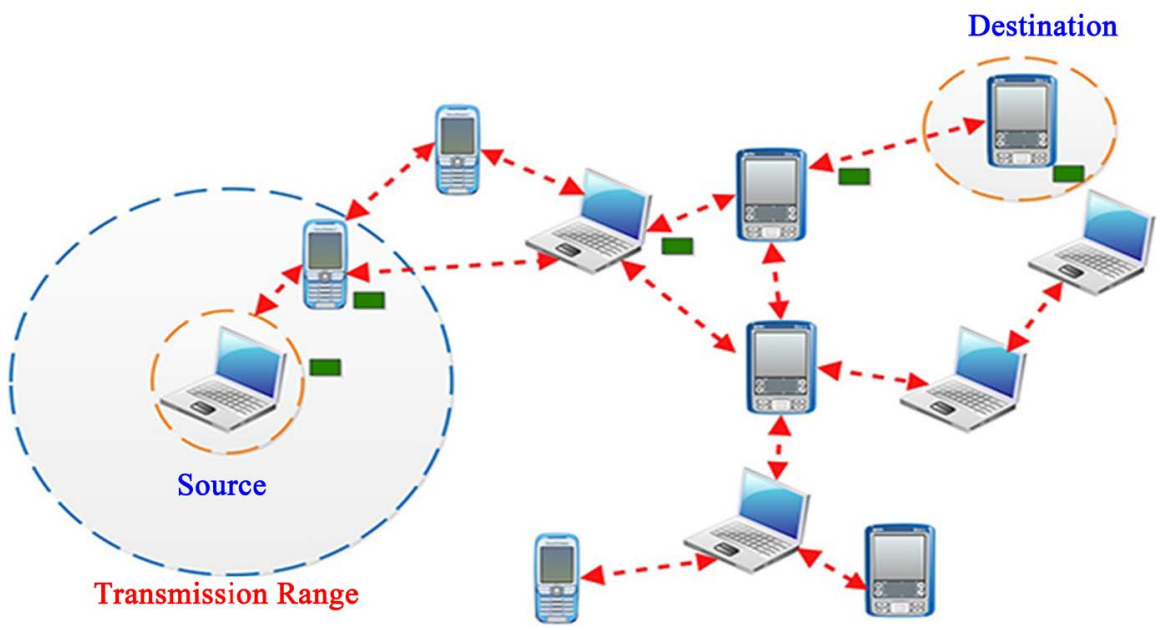

Figure 1. MANET Environments [4]. 
Young-Bae Ko [5] proposed two Directional MAC (DMAC) schemes that are suitable for the IEEE 802.11 based on ad hoc networks using directional antennas to reduce collisions in the network. To get physical location information, each node uses the Global Positioning System (GPS). DMAC scheme 1 utilizes a directional antenna for sending the Ready to Send (RTS) frames in a particular direction, whereas Clear to Send (CTS) frames are transmitted in all directions. DMAC scheme 2 is proposed to reduce the probability of collisions between control frames. In this new scheme there are two types of RTS frames. Directional RTS (DRTS) and Omni-directional RTS (ORTS) are used, if none of the directional antennas at node $\mathrm{X}$ and blocked then node $\mathrm{X}$ will send ORTS and vice versa.

A Multi-hop RTS MAC (MMAC) scheme is introduced in [6] to exploit the higher transmission gain of directional antennas for transmission on multi-hop paths. The MMAC scheme can be considered an enhancement of the DMAC protocol. MMAC could not solve the problems of deafness and hidden terminals, but it could compensate for the negative impact resulting from those problems, and therefore lead to improvement in performance.

A new carrier sensing mechanism called Directional Virtual Carrier Sensing (DVCS) is designed in [7] for MANET using directional antennas. DVCS supports not only directional transmission but directional reception. It does not need specific physical configuration of directional antennas and external devices. Instead of relying on additional GPS devices to locate each node, DVCS only needs minimum information on Angle of Arrival (AoA) and antenna gain for each signal from an underlying physical device.

Jian Shen et al. [8] they evaluated the most recent protocols proposed so far in the literature over directional antennas in terms of several performance metrics such as antenna models, MAC protocols, maximum antenna gain, and number of directional antenna beams, directional neighborhood, multipath support, network throughput, end-to end delay, and routing overhead. Depending on these performance metrics, directional AODV routing protocol has bad network throughput, long end-to-end delay, and high routing overhead. According to this paper, there is no comprehensive study of routing protocols using directional antennas.

So, the main objective of this paper is to investigate new methods which modify AODV routing protocol incorporating directional antenna with multiple network interfaces for improving MANET performances in wireless communication system. That is the reason a lot of research is still being conducted in the design and implementation of directional antenna for routing protocols in MANETs. Figure 2(a) and Figure 2(b) shows the Omni-directional and directional antenna signal transmission respectively.

\section{Research Problem}

The current MANET communication system incorporating Omni-directional 


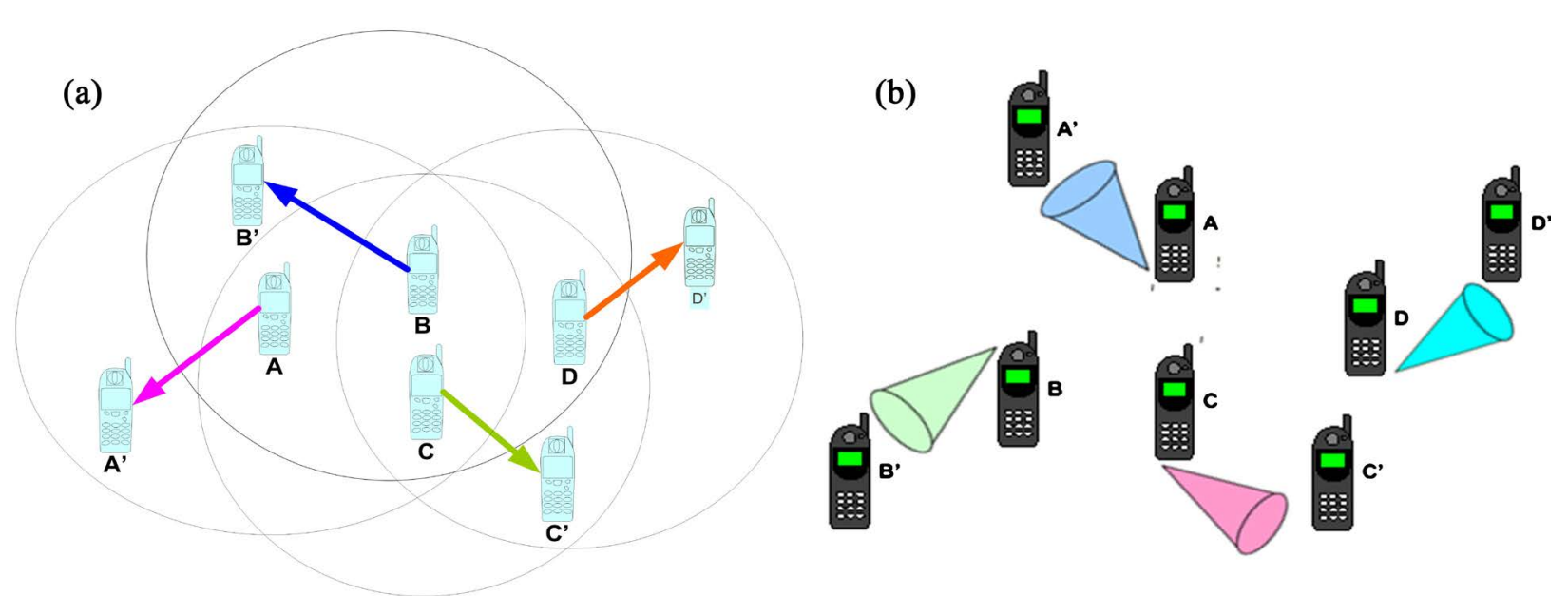

Figure 2. (a) Omni-directional antenna transmission transmission; (b) Directional antenna transmission.

antenna that used the medium much more efficiently and power ineffectiveness since it is radiating radio energy in all directions. The broadcast method of Omni-directional antenna is one of the key problems for simultaneous transmissions. It generates low throughputs and limits the spatial reuse of the shared wireless medium in MANETs. To overcome the problem of this antenna type, integrating directional antenna alleviate wastage of channel resources and transmission of radio energy in unnecessary directions. It is also significantly improves spatial reuse of the wireless channel by permitting several nodes to communicate at the same time without interfering with each other. Thus, significantly increases the capacity of the entire networks. In MANET, the MAC protocols equipped with directional antennas subjected to different problems such as hidden terminal, deafness, route discovery and routing overhead problems when working with current routing protocols [9] [10]. This motivates many research groups to address these problems by studying several techniques for MAC and routing protocols.

A number of researchers were proposed directional antenna for MANETs [8] [11] [12] [13] [14] [15]. But, most of the works on the study of directional antennas for MANET are concentrated on the MAC protocols. They try to implement directional antennas and incorporate it with routing protocols to enhance the performances of the networks. Until now, however, there is no comprehensive study of routing protocols using directional antennas. That is the main reason a lot of research is still being conducted in MANET to increase its performances in different application areas.

\section{Overview of IEEE 802.11 Architecture and Protocols}

The IEEE 802.11 MAC protocol is the standard wireless LAN's (Figure 3). In wireless networking it has two different ways of operation modes [16]. Ad hoc mode or Independent Basic Service Set (IBSS), which allows peer to peer communication between mobile nodes. These mobile nodes should be in the range of each other in order to communicate [16]. The IEEE 802.11 protocol specifies 


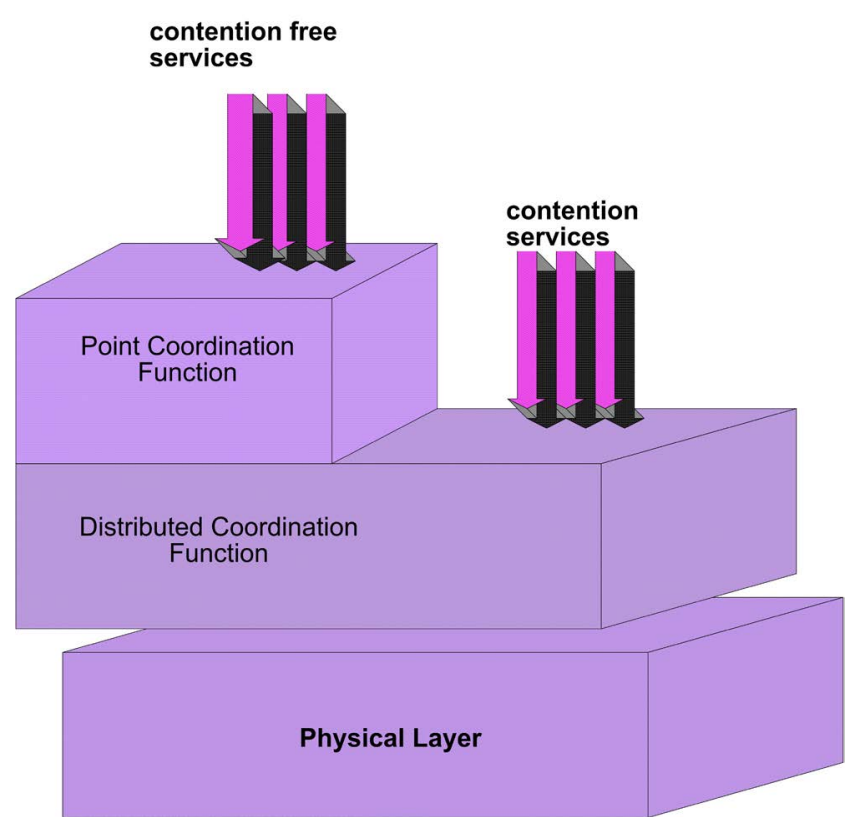

Figure 3. The EEE 802.11 MAC architectures [18].

both the MAC layer and the Physical Layer. The MAC layer provides two different types of services. A contention-based service provided by the Distributed Coordination Function (CDF), and a contention-free service offered by Point Coordination Function (PCF). DCF is based on Carrier Sensing Multiple Access with Collision Avoidance (CSMA/CA). Using CSMA/CA, when a station wants to transmit, it senses the channel. If the channel is idle, it starts to transmit. If the channel is not idle, the sender defers until the channel gets idle and then starts transmitting. When a collision arises, the station involved waits a random time, using the back off algorithm, and then tries again. This used for MANETs. The PCF is implemented on top of DCF. The PCF defined by IEEE 802.11, allows the access to the medium with different priority access. This access method uses a Point Coordinator (PC), which operates at an access point; therefore, PCF operates only in infrastructure-based networks. Note that, the PCF service is not used in MANET since the stations needs access points to communicate each other's [16] [17].

\section{AODV Routing Protocol}

AODV routing protocol is a modified version of both Destination-Sequenced Distance Vector (DSDV) and Dynamic Source Routing (DSR). Because it borrows the basic on-demand mechanism of route discovery and route maintenance from DSR protocol, and the use of hop-by-hop routing, sequence numbers, and periodic beacons from DSDV protocol. The AODV protocol is loop-free and avoids the count-to-infinity problem by the use of sequence numbers. AODV protocol uses a simple request-reply mechanism for route discovery. When a node desires to send a packet to destination, it checks its routing table to determine if it has a current route to the destination. If yes, forwards the packet to 
next hop node. If No, it initiates a route discovery process. AODV uses routing table. This route table is used to store the destination and next-hop IP addresses as well as the destination sequence number. Associated with each routing table entry is a lifetime, which is updated whenever a route is used. Routing in AODV is carried out by the process of route discovery and route maintenance. AODV uses three control messages to obtain and maintain routes. For delivering packets to destination nodes, AODV source node first broadcasting Route Request (RREQ) to the neighbors' nodes. Then the reverse path is used to forward Route Reply (RREP) unicast back to the source. If a node is unable to forward packet, it generates a Route Error (RERR) message. When the originator node receives the RERR, it initiates a new route discovery for the given route [11] [19] [20] [21]. Figure 4 shows the broadcast of the RREQ through the network and the path taken by the RREP from the destination to the source node.

\section{Overview of Directional Antenna}

An antenna in a communications system is a kind of the port through which Radio Frequency (RF) energy is radiated from the transmitter to the outside space for transmission purposes, and in reverse, from the outside space to the receiver for reception purposes [22]. With the fast growth of mobile communication technology, capacity has become one critical issue. The current Omni-directional antennas radiate or receive energy equally in all directions. Because of this antenna, transmitted radio frequency energy in all direction bounds network coverage area, power saving and concurrent communications in MANET. During setting up MANET, we can use different types of directional antennas depending on their operating principle. Switched beam antennas form

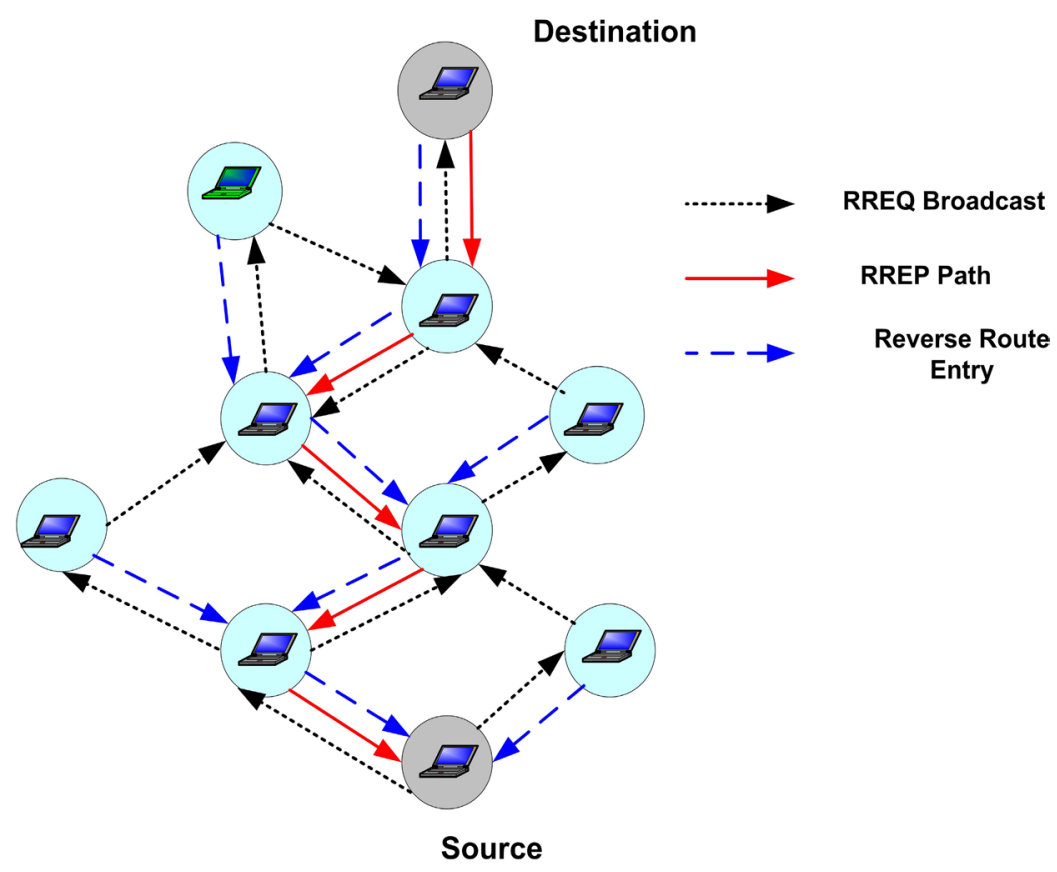

Figure 4. Broadcasts of the RREQ and route discovering mechanisms [11]. 
a finite number of fixed beams in predefined particular directions. This antenna systems detect signal strength, select from one of several predetermined beams that achieves the highest SINR (Signal to Interference and Noise Ratio), and switch from one beam to another as the mobile moves throughout the sector. Adaptive array antenna is the most advanced directional antennas using advanced signal-processing algorithms [22]. It has the ability to effectively locate and track several types of signals to dynamically minimize interference and maximize intended signal reception [22] [23] [24] [25]. Figure 5(a) and Figure 5(b) shows the switched beam and adaptive array antenna in communication system respectively.

\section{Related Works}

This section presents a brief overview on the most relevant works of routing protocols incorporating with directional antenna have been done by different researchers.

M.shyam Sundar et al. [11] they proposed directional based routing protocol (i.e. AODV) in order to increase the performances of MANETs using directional antenna by avoiding interference from nodes hops away, whereas achieving power successfully. The improved version of AODV routing protocol which is based on, the stability of the path, not sending video and data packets together at the same time to discover its routes. It sends it independently at different interval of time. For transmitting video and packets, they used 802.11e MAC which gives priority to video packets more than data packets. The modified AODV based on stability of the path can adapt itself dynamically to cope up with the mobility of network and calculate the stability of the path based on the received signal strength and inserting of some additional fields in RREQ/RREP packets. To investigate the best route, the authors chooses three metrics such as hop count, power budget and overlaps between adjacent beams for transmission of real time data (i.e. voice and video) in the route discovery process. According to this paper AODV shows the low throughputs.

Mandeep Singh et al. [12] they evaluated the performances of three routing protocols AODV, Geographic Routing Protocol (GPR) and Optimized Link State Routing (OLSR) using directional antenna in MANETs. To explore the effects of using directional antenna in MANETs, they compare the performances

(a)

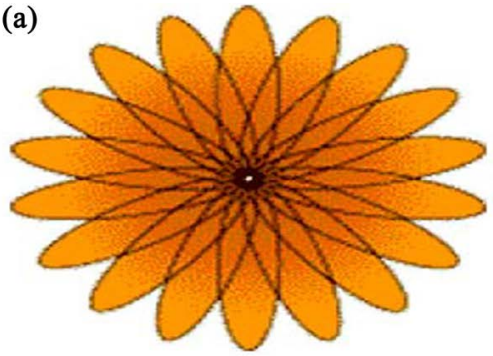

(b)

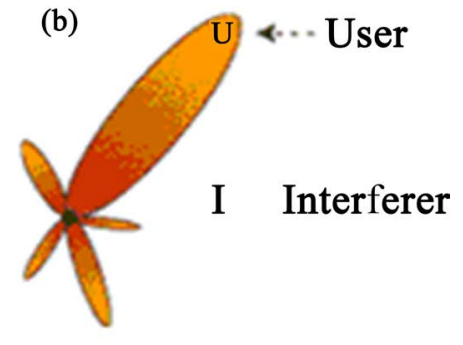

Figure 5. (a) Switched d beam antenna patterns [25]; (b) Adaptive array antenna patterns [25]. 
of routing protocols by using the same simulation scenarios for both omnidirectional and directional antenna. For designing the antenna patterns they used some mathematical and trigonometric equations. They implemented one omnidirectional antenna for node model and two directional antennas for node model; one directional antenna at transmitter node and the second at the receiver node in case of directional antenna. According to this study incorporating directional antennas into MANETs offers better network performance than omnidirectional antennas.

Ankit Jindal et al. [13] they proposed a MAC protocol with directional antenna based on a circular directional Ready to Send (RTS) and scan the area around the transmitter and informing the next nodes for the communication to reduce the hidden and deafness problem caused by directional antennas. For the evaluation purpose they used three different types of antennas. These antennas are omnidirectional, switch beam and steerable antennas. From the experimentation results, AODV provides highest values for average jitter and end to end delay and consume less energy among others routing protocols depends on different antenna types.

Rajesh Kumar Yadav et al. [14] they used three types of different sectorized antenna system for broadcasting the RREQ in selected beams. This sector antenna systems are: 3 sector antennas, 4 sector antennas, and 8 sector antennas systems. According to this paper nodes do not transmit the RREQ to the sectors from where it receive the RREQ using 3 sector antenna or 4 sector antenna, while in 8 sector antenna, the sectors of the source node of RREQ and its adjacent sectors do not receive RREQ packet. During experimentation, they reported $35 \%$ reduction in routing overhead when compared with conventional blind flooding the RREQ on several standard regular models using selective flooding for RREQ broadcast.

Arvind Kumar et al. [15] they proposed adaptive routing scheme that use directional antenna for route selection in the best way. To select the best routes among others, the authors used correlation factor and propagated hop count. They discovered the effect of directional antenna on multipath routing and compare its effectiveness with omnidirectional antenna. The performances of multipath is high when the number of communication or connections is low, but when the number of simultaneous communication increases or the number of traffic flow is high during concurrent communication, the performance of multipath routing degrades as compared to single-path transmission.

Jian Shen et al. [8] they evaluated the performances of different routing protocols such as directional DSR, directional AODV, Energy Efficient Directional Routing (EEDR), Directional Antenna Multipath Location Aided Routing (DA-MLAR) and Multipath Directional Antenna Ad Hoc Routing (MDAR) so far in terms of several performance metrics. Depending on these performance metrics, directional AODV routing protocol has bad network throughput, long end-to-end delay, and high routing overhead. 
In general, most of the researches were done in MANET on different topics to improve the performances of the networks. But, still now there is no comprehensive study of routing protocols using directional antennas and multiple network interfaces. Hence, this research was done for solving these problems by applying directional antenna and multiple network interfaces in MANET routing protocols.

\section{Implementation Tool}

NS2 is an object-oriented, discrete event driven network simulator developed at UC Berkely written in $\mathrm{C}++$ and $\mathrm{OTcl}$ (Tcl script language with Object-oriented extensions). It implements network protocols such as TCP and UPD, traffic source behavior such as FTP, Telnet, Web, CBR and VBR, router queue management mechanism such as Drop Tail, RED and CBQ, routing algorithms such as Dijkstra, and more. NS2 also implements multicasting and some of the MAC layer protocols for LAN simulations. NS2 includes a tool for viewing the simulation results, called NAM (Network Animator). NAM is a Tcl/TK based animation tool for viewing network simulation traces and real world packet trace data. NS2 has a rich library of network and protocol objects [26]. Figure 6 shows the NS2 directory structures.

\subsection{NS2 Directory for Directional Antenna Model Implementation}

A simple directional antenna module for NS2 is available. The antenna is modeled as switched beam antenna. The antenna returns a gain of 1 multiplied by the solid angle, if the node falls inside the cone of coverage formed. The directional antenna module takes its input from an antenna file which is expected to be in the NS_ANTENNA_FILE environment variable. The antenna patch must be added to the NS2 after it is installed and the patch is necessary to support

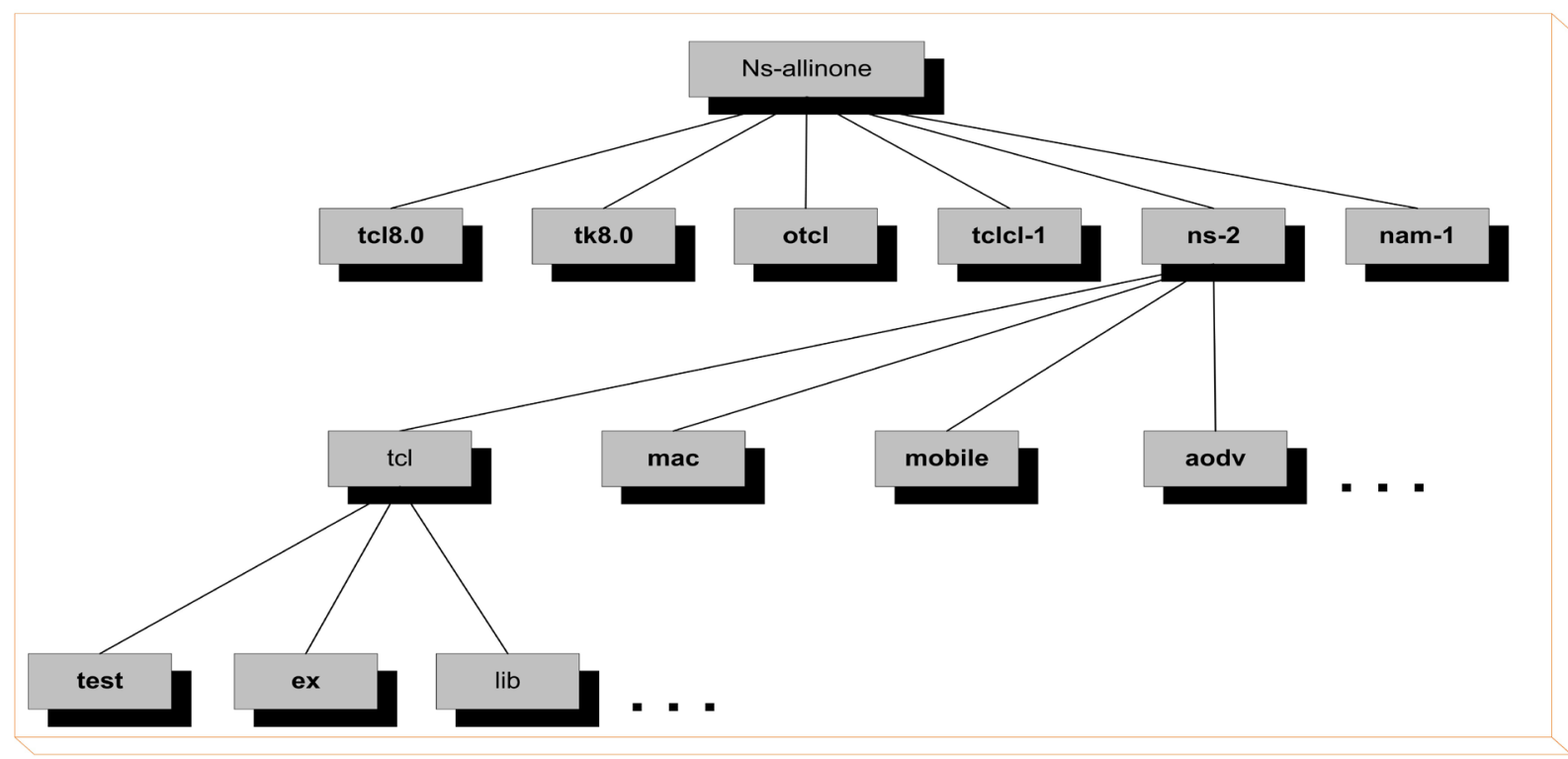

Figure 6. NS2 directory structures [26]. 
multiple wireless interfaces [27] [28]. Figure 7 shows the place where the patch of directional antenna is implemented in NS2 directory structures and where a change is occurs.

\subsection{NS2 Directory for Multiple Interface Model Implementation}

The fast development of the IEEE 802.11 technology has make better the sharp decrease of the corresponding products' prices and therefore, their presence is each day more and more common. This has gathered the interest of the NS2 community, since a lot of researchers are willing to extend their simulation models to incorporate multiple interfaces. To add the multiple interfaces model in mobile node architecture, we implement it at some NS2 directory structures like routing protocol, TCL/LIB, MAC layer and mobile nodes. For routing protocol, this model used to manage multiple interfaces, create multi-interfaces nodes and adding some random time to avoid collisions during the RREQ packet transmissions from source node to destination node. In NS2 library the multi-interfaces module implemented in ns-lib.tcl and ns-mobilenode.tcl for allows different number of interfaces per node, add an interface (channel) to a node and getting the number of interfaces from the simulator object. In mobile node, [.cc \&.h], it connects the multiple interfaces to appropriate channel number for managing the nodes of one particular channel. At MAC layer it needs to identify the interface which a message was received through. This makes the MAC layer for the correct handling of multiple interfaces by the routing protocol [27] [28]

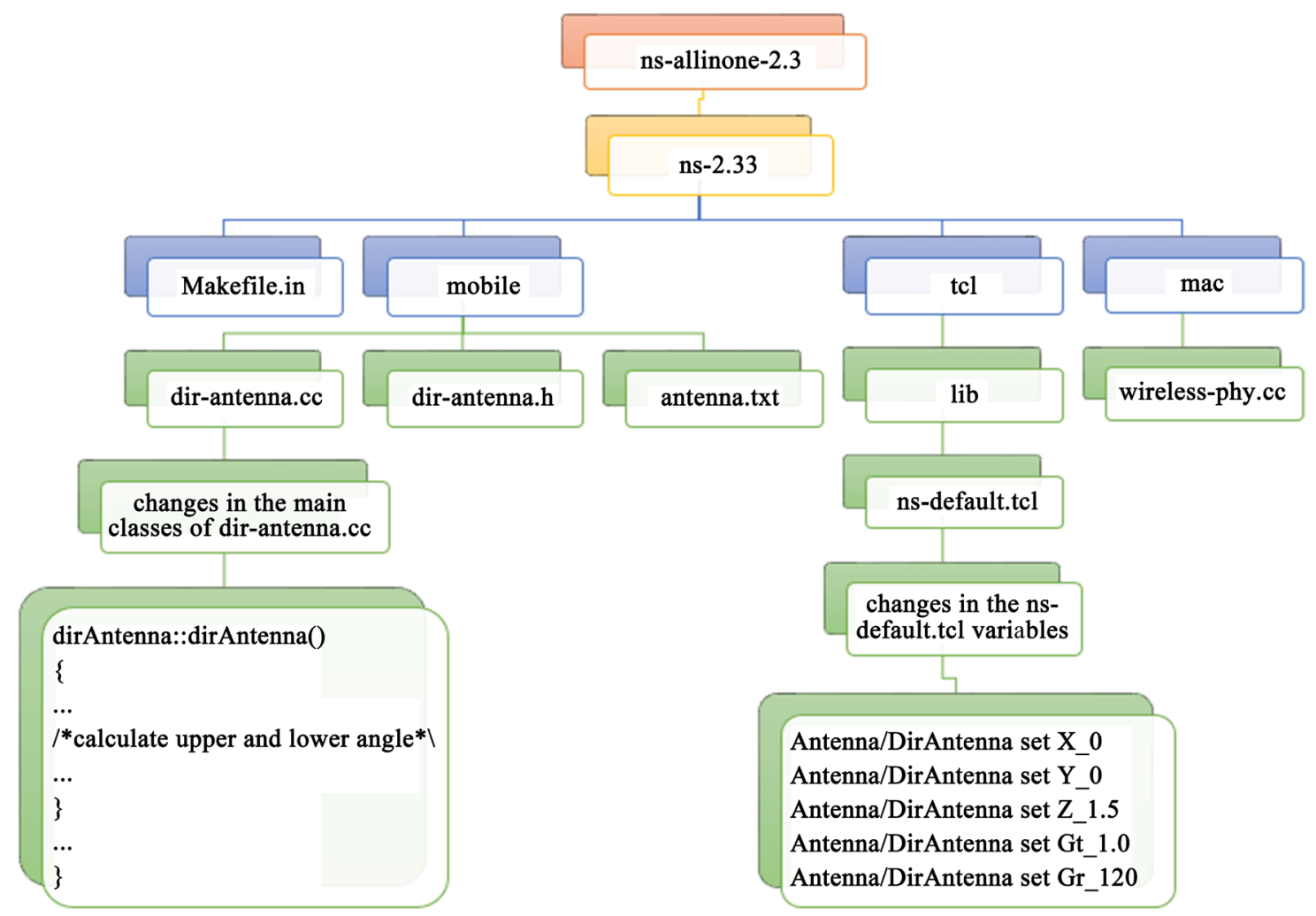

Figure 7. NS2 directory Structure used for implementing directional antenna model. 
[29]. Figure 8 illustrating the place where the multiple interfaces implementation at some NS2 directory structure and changes in NS2c++ codes.

\section{Simulation Results and Discussions}

\subsection{Performance Metrics}

This section presents all the quantitative parameters which were measured and analyzed in these simulation scenarios.

\subsubsection{Throughput}

It is the phenomena from which we calculate the average rate of successful data packet from received at destination. It is measured in bits second, sometimes in data packet per second, in Kbps, Mbps and Gbps [13].

\subsubsection{End-to-End Delay}

It is the phenomena from which we calculate the difference between send time and receive time when data send from source to destination node. It is measure in seconds. It includes the route discovery delay, the queuing delay at each intermediate node, the contention delay at MAC layer and the transmission delay for each hop [13].

\subsection{Random Topology Scenario}

A random topology was used in this simulation model. Each node is arranged at random in square area dimensions of $100 \mathrm{~m} \times 100 \mathrm{~m}$. The source and destination nodes were randomly chosen. In this simulation model, we evaluated the performances of AODV routing protocol using omnidirectional and directional

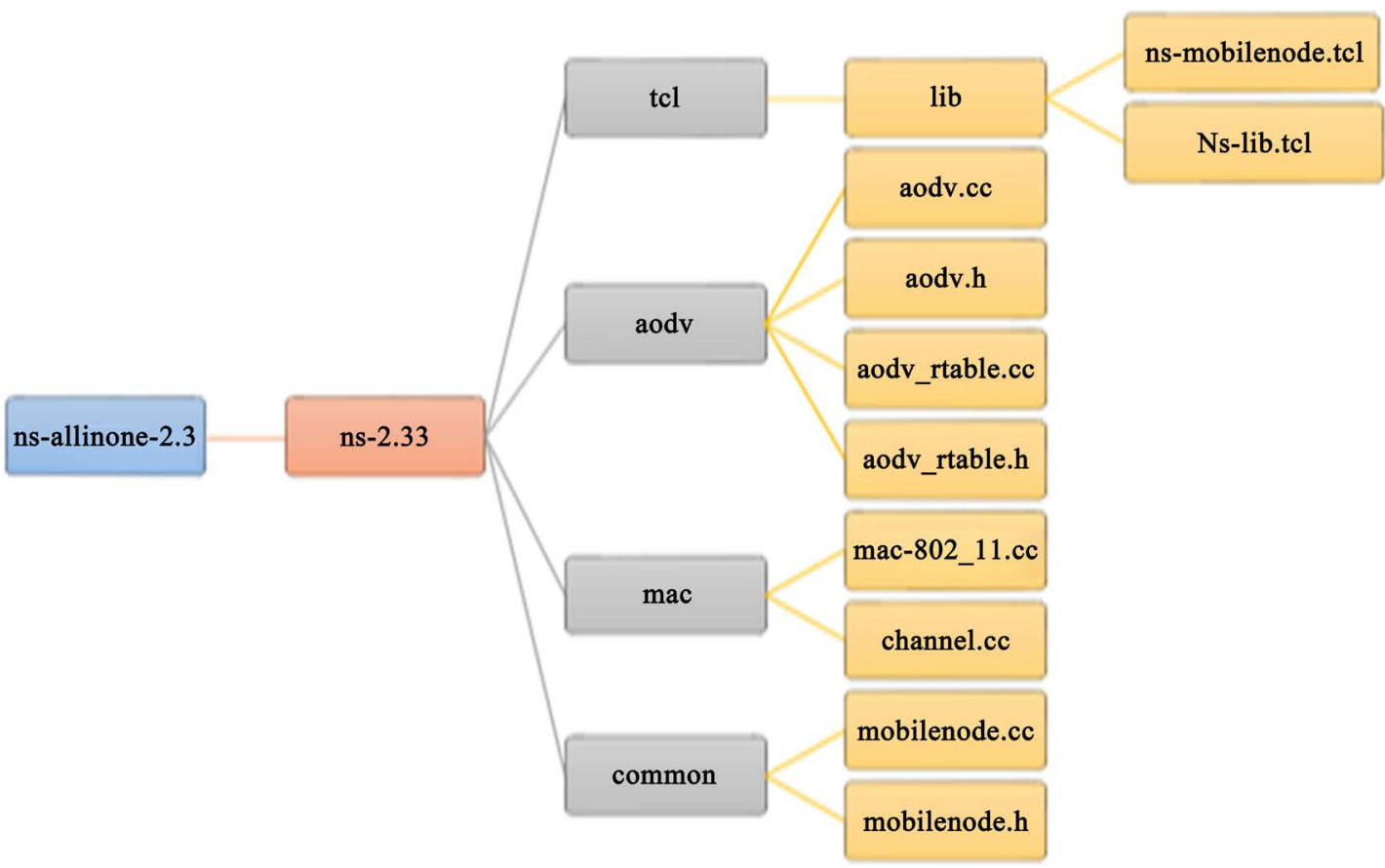

Figure 8. NS2 directory structure used for implementing multiple interfaces model. 
antennas by increasing communication paths. Each simulation is performed for duration of 150 seconds. Table 1 summarizes the parameters used in our simulation.

The throughput of different number of connections is presented in Figure 9. The throughputs of AODV routing protocol with directional antenna outperforms the throughputs of AODV routing protocol with Omni-directional antenna. Because, using directional antenna in mobile communication offers good throughputs during concurrent transmission by sending RREQ to the destinations through specified angles. This avoids blind RREQ in the network that creates more data collisions. But, the AODV with Omni-directional antenna provides fewer throughputs as seen in Figure 9. Since it broadcasts the RREQ in all directions, collision of packets are created and this lead to reduce the throughputs of AODV routing protocol. During single number of flows, the throughputs of both routing protocols have no significant differences. So, the single communication path does not affect the performances of AODV with Omni-directional antenna when compared with multiple numbers of connections. However, in higher simultaneous communications flows, the throughputs of the omnidirectional AODV go down whereas the throughputs of directional AODV still increasing from connection 1 to 10 as shown in Figure 9.

This indicates that using Omni-directional antenna in dense communication

Table 1. Parameter values for simulation.

\begin{tabular}{|c|c|}
\hline Parameters & Values \\
\hline Number of nodes & 10 \\
\hline Type of channel & Wireless channel \\
\hline Area & $100 \times 100$ meters \\
\hline Mac layer protocol & MAC IEEE802.11 \\
\hline Packet size & 1024 bytes \\
\hline Directional transmission range & 250 meters \\
\hline Carrier sense threshold & 550 meters \\
\hline Path-loss model & Two ray ground \\
\hline Directional antenna gain & $10.0 \mathrm{~dB}$ \\
\hline Simulation Time & 150 seconds \\
\hline Node mobility & Static \\
\hline Number of flows & $1,2,4,6,8,10$ \\
\hline Routing protocol & AODV \\
\hline Beam width & 120 degrees \\
\hline Directional number of interfaces & Different number of interfaces per node \\
\hline Antenna type & Directional antenna, omnidirectional antenna \\
\hline Antenna model & Switched beam \\
\hline Traffic model & FTP \\
\hline
\end{tabular}




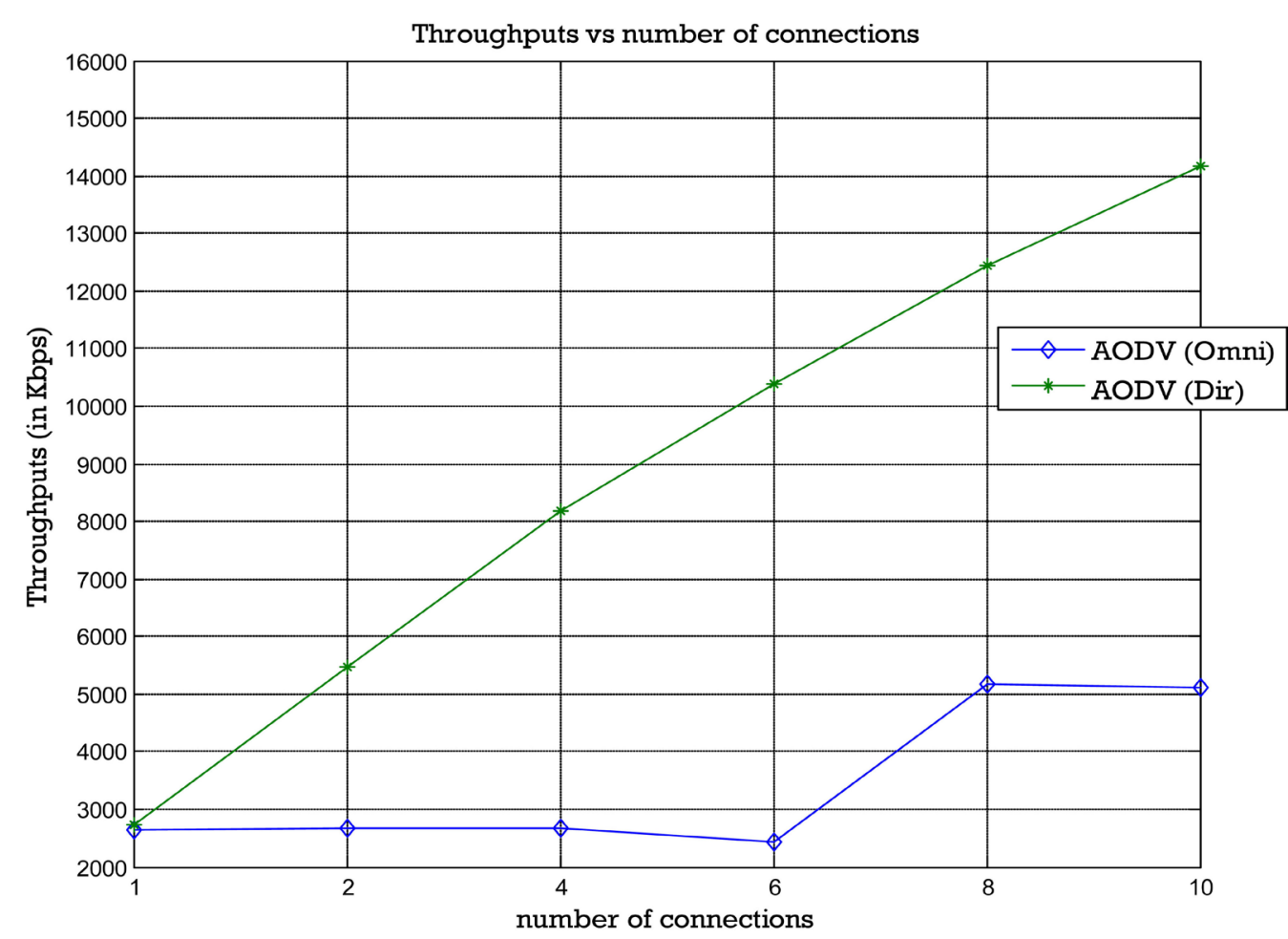

Definition: AODV $($ Omni $)=$ Omni-directional AODV; AODV $($ Dir $)=$ Directional AODV.

Figure 9. Comparing throughputs of AODV with directional and omnidirectional antenna.

environment deteriorates the network performances. Increasing number of connections increases the throughputs. Since, the sum of each individual connection provides the total throughputs of the number of connections in the network. From Figure 9, the throughputs of Omni-directional AODV increases from connection 1 to 4 and 6 to 8 . But, from connection 4 to 6 the throughputs of this routing protocol is decreased. The reason is the way of communication flow, distance and number of hops between the source nodes to destination nodes. This creates high collisions and delay. For example, the packet dropped for connection 1, 2 and 4 are 20,53 and 171 respectively and 940 in case of connection 6. For connection 8 , the dropped packets are 670 . But, connection 8 has less dropped packets than connection 6 . This indicates that there are high collisions for connection 6 . This could results in decreasing the throughputs between connections 4 to 6 .

Generally, increasing number of connections increases the throughputs of AODV up to some levels for both directional and Omni-directional AODV routing protocols. But, increasing number of connections above network capacity decreases the throughputs since it creates more collisions. So, using directional antenna in wireless communications improves the throughputs of routing protocols. It increases the throughputs of AODV almost by $50 \%$ comparing with the throughputs of Omni-directional AODV as shown in Figure 9 in higher connections.

The end to end delay is presented in Figure 10. The end to end delay for 


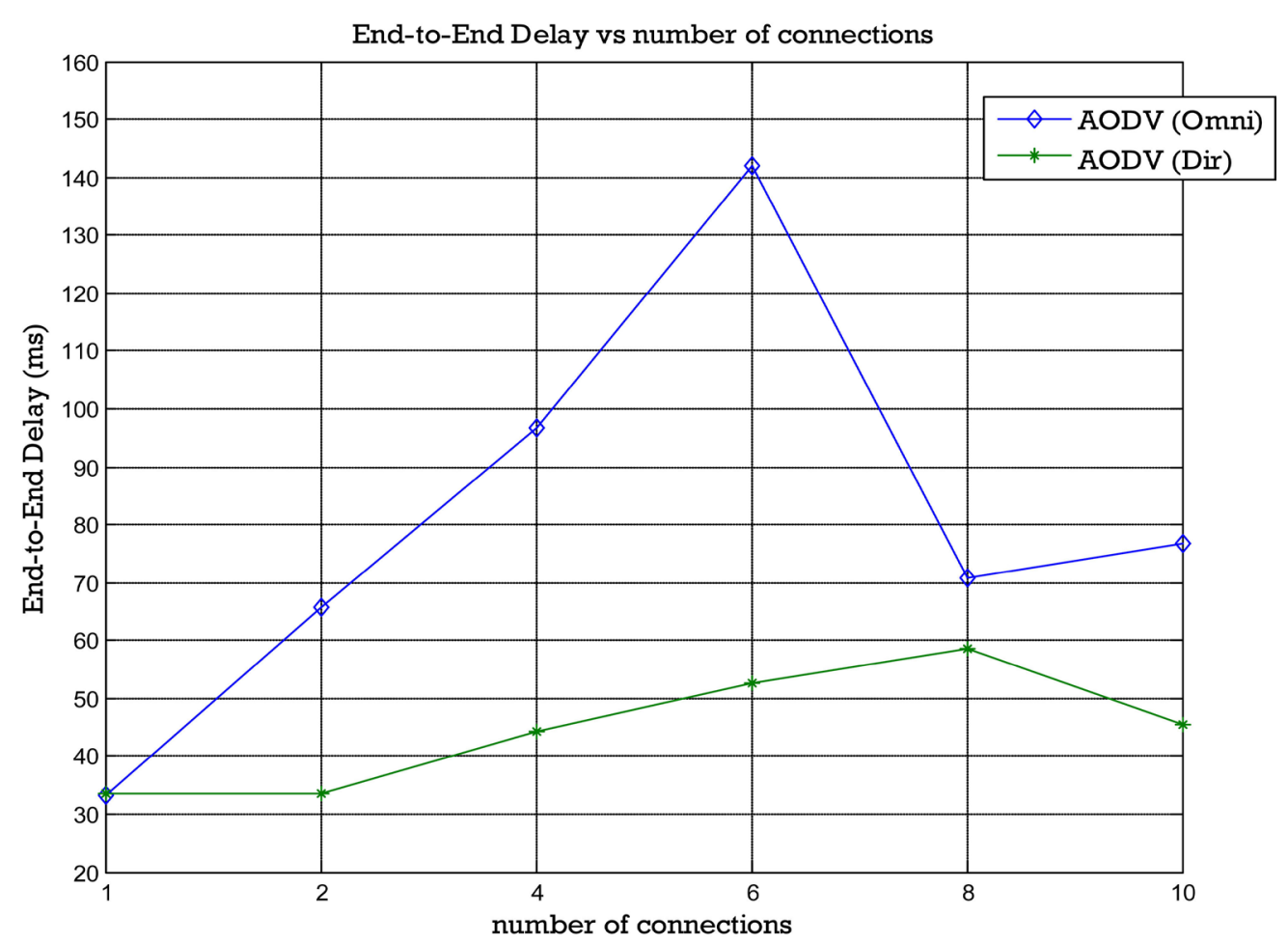

Figure 10. Comparing end to end delay of AODV with directional and omnidirectional antenna.

directional AODV outperforms the Omni-directional AODV. For single connection, the end to end delay for both routing protocols is the same. Because, single connection could not create high collision as compared to more communication flows. In high communication paths, problems like exposed terminal, hidden terminal and deafness could happen. This makes packet collisions and high retransmission of data that increases end to end delay in the network. From Figure 10, the end to end delay of directional AODV routing protocol is the same for connection 1 and 2 while the end to end delay of Omni-directional AODV increasing. The reason is the directional AODV uses more flexible network interfaces that associated to different channels for connection 1 and 2 in addition to directional beams. This decreases packet collisions by improving the RREQ mechanisms. However, from connection 2 to 8 the end to end delay of this protocol is increasing with number of connections. Because, while the number of communication paths increases, end to end delay increases. From connection 8 to 10 , the end to end delay for directional AODV is decreasing. This is depends on communication paths and network topology. The end to end delay for Omni-directional AODV increases from connection 1 to 6. Because, Omni-directional AODV is increases end to end delay during simultaneous transmissions. Since it create high collisions in high communication paths. Comparing with connection 4 and 6, connection 8 and 10 has low end to end delay in case of Omni-directional AODV. This depends on distances and number of hops between source to destination node and network topology. According to Figure 10, directional AODV routing protocol has lower end to end delay 
than Omni-directional AODV protocol in all cases.

\subsection{Grid 6 × 6 Scenario}

The grid scenario is formed by 36 nodes placed in a square forming 6 rows and 6 columns. Each node is separated 100 meters from its neighbors. The carrier sense threshold is 550 meters and the transmission range is 250 meters for each node in case of both directional and omnidirectional antennas. Table 2 summarizes the parameters for simulation.

This simulation scenario consists of one five-hop connection: the connection is from node 18 to node 23. The throughput of directional AODV is higher than the throughput of Omni-directional AODV protocol. The Omni-directional AODV has high end to end delay as seen in Figure 11. Because, the omnidirectional AODV broadcast the RREQ in all direction. This decreases the amount of power that the packet required to reach destination node. This results in high end to end delay and provides low throughputs than directional AODV.

As seen in Figure 12, this scenario consists of two one-hop connections: one connection is from node 2 to node 3 and the other from node 4 to 5 . In this simulation scenario also the throughput of directional AODV outperforms the omnidirectional AODV. In both routing protocols throughput is increased when compared with the simulation scenario in Figure 11. But, the end to end delay of the two routing protocol is less than end to end delay of the simulation scenario

Table 2. Parameter values for simulation.

\begin{tabular}{cc}
\hline Parameters & Values \\
Number of nodes & 36 \\
Node placement & Grid \\
Grid size & 100 meters \\
Type of channel & Wireless channel \\
Terrain size & $100 \times 100$ meters \\
Mac layer protocol & MAC IEEE 802.11 \\
Packet size & 1460 bytes \\
Directional transmission range & 250 meters \\
Carrier sense threshold & 550 meters \\
Path-loss model & Two ray ground \\
Directional transmission gain & 10.0 dBm \\
Simulation Time & 150 seconds \\
Mobility model & None \\
Routing protocol & AODV \\
Directional beam width & 120 degrees \\
Number of interfaces & 2 \\
Antenna type & Directional antenna, omnidirectional antenna \\
\hline
\end{tabular}


in Figure 12. The reason for this is the distance and number of hops between sources to destinations.

As seen in Figure 13, this scenario consists of two one-hop connections in the opposite direction: one connection is from node 1 to node 0 and the other from node 2 to 3. In this simulation scenario the throughput of directional AODV outperforms the Omni-directional AODV. In both routing protocols throughput is increased when compared with the simulation scenario in Figure 11. But, the

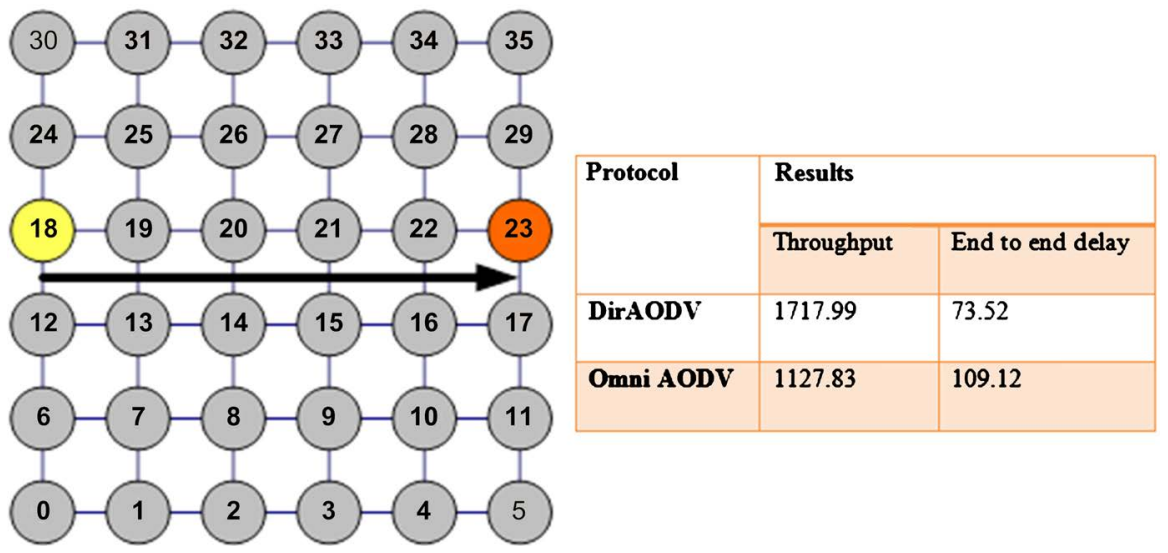

Figure 11. Simulation scenario for one five-hop connection.

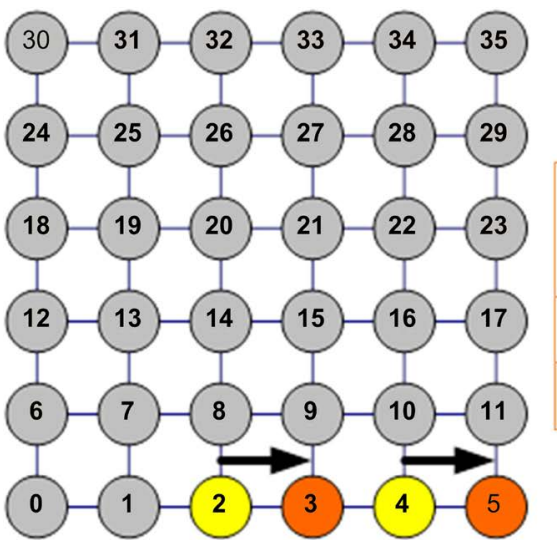

\begin{tabular}{|c|c|c|}
\hline \multirow[t]{2}{*}{ Protocol } & \multicolumn{2}{|l|}{ Results } \\
\hline & Throughput & End to end delay \\
\hline DirAODV & 6888.67 & 36.67 \\
\hline Omni AODV & 3399.11 & 72.38 \\
\hline
\end{tabular}

Figure 12. Simulation scenario for two one-hop connections in the same direction.

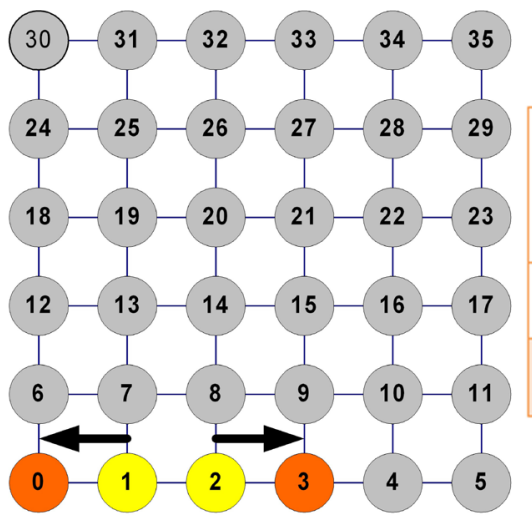

\begin{tabular}{|c|c|c|}
\hline \multirow[t]{2}{*}{ Protocol } & \multicolumn{2}{|l|}{ Results } \\
\hline & Throughput & End to end delay \\
\hline DirAODV & 6889.05 & 36.67 \\
\hline Omni AODV & 3399.12 & 72.38 \\
\hline
\end{tabular}

Figure 13. Simulation scenario for two one-hop connections in the opposite direction. 
same with the simulation scenario in Figure 13. Only the difference between the simulation scenario in Figure 12 and Figure 13 is the direction of connection. In both cases, they have equal distance and single hop from source to destination nodes and the source nodes are within the range of each other's. So, the two simulation scenarios have the same throughput and end to end delays.

As seen in Figure 14, this scenario consists of two five-hop connections in the different direction: one connection crosses a row in the $6 \times 6$ from node 0 to node 5 , and another connection crosses a column from node 0 to node 30 . Both paths consist of five hops. In this case, this simulation scenario has low throughput and high end to end delays when compared with the simulation scenarios in Figure 12 and Figure 13. Since, this simulation scenario has different number of hops and way of communication paths.

As seen in Figure 15, this scenario consists of six simultaneous connections in the same directions. All paths consist of five hops. During simultaneous communications, the throughput of directional AODV routing protocol outperforms the omnidirectional AODV. The end to end delay of directional AODV also less than Omni-directional AODV. As compared with other simulation scenarios in Figures 11-14, the two routing protocols in Figure 15

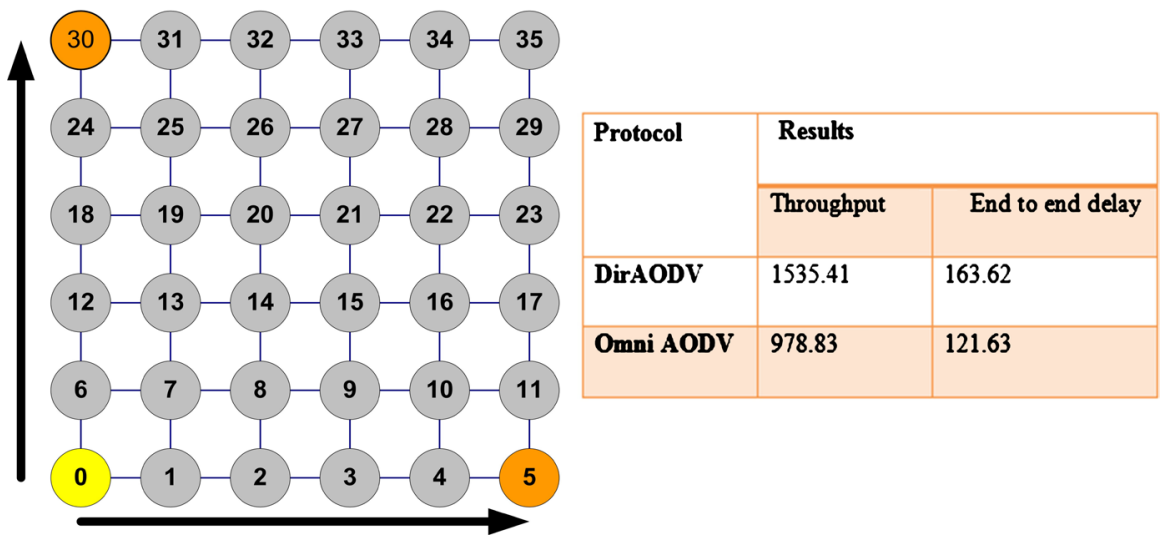

Figure 14. Simulation scenario for two five-hop connections in the different directions.

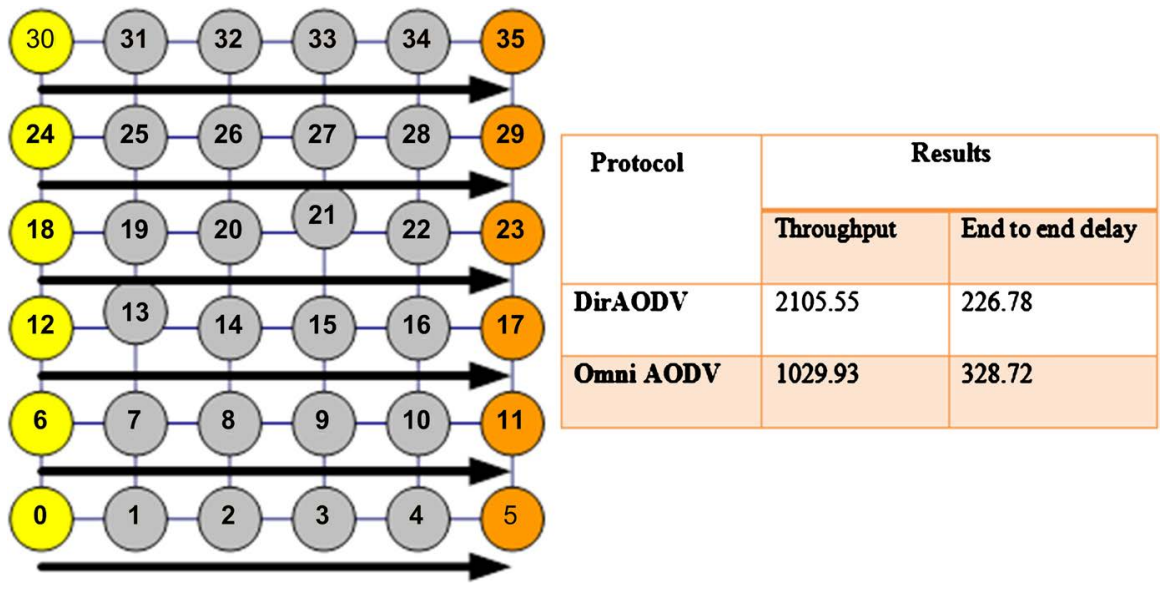

Figure 15. Simulation scenario for six simultaneous connections. 


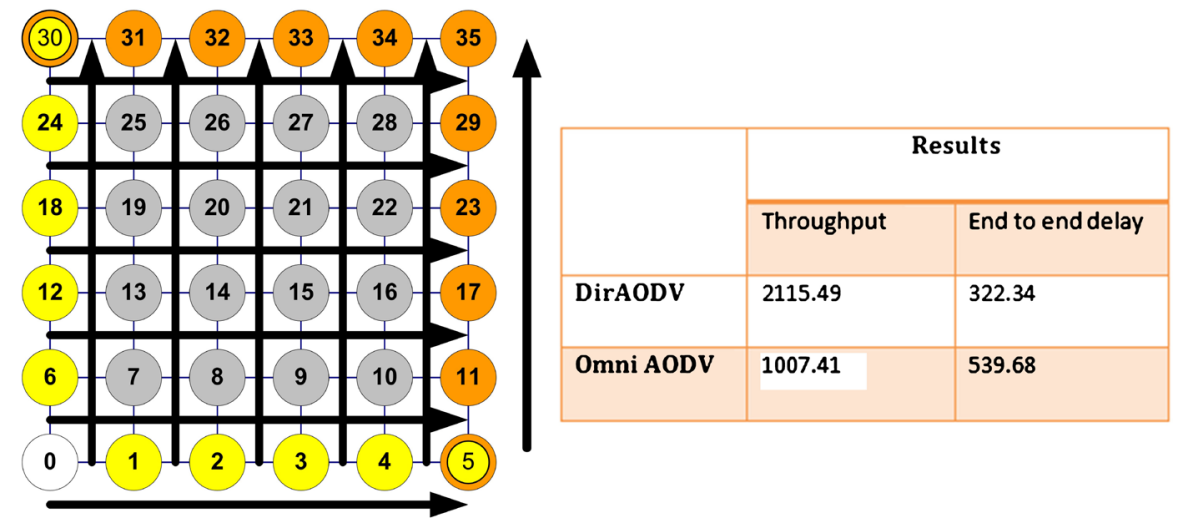

Figure 16. Simulation scenario for twelve simultaneous connections.

has less throughput and high end to end delay. Because, this modified versions of AODV routing protocol provides high spatial reuse and increase ranges that reduces packet collisions.

In the simulation scenario Figure 16, we increase the number of simultaneous communication paths from six to twelve. All paths consist of five hops. All nodes in the first column and sixth row are source nodes. All nodes in the first row and sixth column are destination nodes. Node 0 connection traverses a row in the $6 \mathrm{x}$ 6 from node 0 to node 5 , and another connection traverses a column from node 0 to node 30 . Both nodes 5 and 30 are sources and destination. In this simulation scenario, the end to end delays for the two protocols are high relating with six simultaneous communications in Figure 15. From this simulation scenario in Figure 16, we concluded that directional antenna has higher capacity of carrying communication paths than Omni-directional antenna.

\section{Conclusion and Future Work}

\subsection{Conclusions}

In this study, the concept of the MANET, AODV routing protocol, and the implementation of smart antenna with multiple interfaces techniques in MANETs were studied. The results show that network performance of directional antennas is not affected much by increasing the communication distances and number of connections in simulation scenarios. The routing performance of directional AODV outperforms omnidirectional AODV in the higher communication paths and in long communication distance. It decreases the hidden terminal and deafness problem and provides high throughputs in the presence of higher number of connections. To evaluate the performance of the routing protocols using different antenna models, this paper used the two well-known network topologies and different performance metrics. Directional antenna outperforms omnidirectional antenna in both topologies. Simulation results show that the proposed technique achieves around 50\% increases over the throughput of the current antenna model. Generally, we recommend that implementing directional antenna in MANETs improves the performances of the wireless communica- 
tion system.

\subsection{Future Work}

The MANET with directional antenna is a hot research area in the communication system. There are many researches going on and many issues that remain to be addressed. There are still many problems that could be left for further studies, such as:

- This paper evaluates only one reactive routing protocol using different antenna models. Other reactive and proactive routing protocols are also an interesting issue that needs to be evaluated.

- In this study work, we did not consider mobility. So, designed directional antenna to appropriately manage the nodes mobility is an open for future research.

- Security is not considered. It is a very important issue in MANETs to be implemented with directional MAC protocol.

- The authors used switched beam antenna system and directional MAC IEEE 802.11. Therefore, considering different directional antenna types and MAC protocols for route discovering is an important issue.

\section{Conflicts of Interest}

The authors declare no conflicts of interest regarding the publication of this paper.

\section{References}

[1] Kaponias, A., Politis, A. and Hilas, C. (2012) Simulation and Evaluation of MANET Routing Protocols for Educational Purposes. 2nd Pan-Hellenic Conference on Electronics and Telecommunications, Thessaloniki, Greece, 16-18 March 2012.

[2] Hussain, M.A., Suresh Varma, P., Satya Rajesh, K., Pathan, H.B. and Sarraju, L.M. (2010) Use of Smart Antennas in Ad Hoc Networks. International Journal of Computer Science \& Information Technology (IJCSIT), 2, 47-54. https://doi.org/10.5121/ijcsit.2010.2605

[3] (2020) Lecture Notes on Mobile Computing. http://www.alljntuworld.in

[4] (2020) Thesis Writer and M. Tech Thesis Project in Bathinda and Patiala.html

[5] Ko, Y.-B., Choi, J.-M. and Vaidya, N.H. (2007) MAC Protocols Using Directional Antennas in IEEE 802.11 Based Ad Hoc Networks. Wireless Communications and Mobile Computing, 8, 783-795.

[6] Choudhury, R.R., Yang, X., Ramanathan, R. and Vaidya, N.H. (2002) Using Directional Antennas for Medium Access Control in Ad Hoc Networks. Proceedings of the 8th Annual International Conference on Mobile Computing and Networking, Atlanta, September 2002, 59-70. https://doi.org/10.1145/570645.570653

[7] Takai, M., Martin, J., Ren, A. and Bagrodia, R. (2002) Directional Virtual Carrier Sensing for Directional Antennas in Mobile Ad Hoc Networks. Proceedings of the 3rd ACM International Symposium on Mobile Ad Hoc Networking \& Computing, Lausanne, June 2002, 183-193. https://doi.org/10.1145/513800.513823

[8] Shen, J., Zheng, W., Wang, J., Xia, Z. and Fu, Z. (2013) Routing Protocols Using 
Directional Antennas in Ad Hoc Networks: A Comparative Review. International Journal of Grid and Distributed Computing, 6, 39-50. https://doi.org/10.14257/ijgdc.2013.6.5.04

[9] Watanabe, M., Mitsuhashi, H., Bandai, M., Obana, S. and Watanabe, T. (2007) Empirical Discussion on Directional MAC Protocols for Ad Hoc Networks Using Practice Smart Antennas. IEEE International Conference on Communications, Glasgow, 24-28 June 2007, 3642-3647.

[10] Wang, J., Zhai, H., Li, P., Fang, Y. and Wu, D. (2009) Directional Medium Access Control for Ad Hoc Networks. Wireless Networks, No. 8, 1059-1073.

[11] Sundar, M.S. and Karthik, N. (2012) Enhancing the Performance of Ad Hoc Routing Protocols Using Directional Antennas. International Journal of Computer Science and Electronics Engineering, 2, 2976-2981.

[12] Singh, M. and Singh, M. (2013) Performance of AODV, GRP and OLSR Routing Protocols in Adhoc Network with Directional Antennas. International Journal of Computer Applications, 83, 12-18. https://doi.org/10.5120/14419-2543

[13] Jindal, A., Singh, C. and Vir, D. (2014) Simulation Analysis of Different Routing Protocols Using Directional Antenna in Qualnet 6.1. International Journal of Advanced Research in Electrical, Electronics and Instrumentation Engineering, 3, 9408-9414.

[14] Yadav, R.K. (2013) Efficient AODV Using Improved Flooding with Sectorized Antenna for Mobile Adhoc Networks. International Journal of Advanced Research in Computer Science and Software Engineering, 3, 922-926.

[15] Kumar, A. and Tripathi, R. (2010) Performance Improvement of Routing Protocol in Ad-Hoc Networks Using Smart Antenna with Balance Load. International Journal of Computer and Electrical Engineering, 2, 996-1000.

[16] Goel, S. (2006) Performance Evaluation of TCP over IEEE 802.11 WLANs. Master Thesis, Sikkim Manipal Institute of Technology (Sikkim), India.

[17] Xu, K., Gerla, M. and Bae, S. (2003) Effectiveness of RTS/CTS Handshake in IEEE 802.11 Based Ad Hoc Networks. Ad Hoc Networks, 1, 107-123. https://doi.org/10.1016/S1570-8705(03)00015-5

[18] Laurea, G.A., Conti, M. and Laurea, E.G. (2004) IEEE 802.11 Ad Hoc Networks: Protocols, Performance and Open Issues. Mobile Ad Hoc Networking, 69-116.

[19] Mbarushimana, C. and Shahrabi, A. (2007) Comparative Study of Reactive and Proactive Routing Protocols Performance in Mobile Ad Hoc Networks. 21 st International Conference on Advanced Information Networking and Applications Workshops (AINAW07), Niagara Falls, 21-23 May 2007, 679-684. https://doi.org/10.1109/AINAW.2007.123

[20] Saini, G.L. and Dembla, D.D. (2013) Modeling, Implementation and Performance Evaluation of E-AODV Routing Protocol in MANETs. International Journal of Advanced Research in Computer Science and Software Engineering, 3, 1221-1227.

[21] Ahmad, I., Ashraf, U., Anum, S. and Tahir, H. (2014) Enhanced AODV Route Discovery and Route Establishment for QOS Provision for Real Time Transmission in MANET. International Journal of Computer Networks \& Communications (IJCNC), 6, 79-87. https://doi.org/10.5121/ijcnc.2014.6207

[22] Solheid, A. (2005) AODV Enhanced by Smart Antennas. Master Thesis, University of Namur, Namur.

[23] Uthansakul, M., Uthansakul, P. and Wongchompa, P. (2010) Performance Evaluation of Automatic Switched-Beam Antennas for Indoor WLAN Systems. School of 
Telecommunication Engineering, Suranaree University of Technology, Nakhon Ratchasima, 782-792.

[24] Gotsis, K.A. and Sahalos, J.N. (2011) Beamforming in 3G and 4G Mobile Communications: The Switched-Beam Approach. In: Maícas, J.P., Ed., Recent Developments in Mobile Communications-A Multidisciplinary Approach, IntechOpen, London, 201-216.

[25] (2005) Smart Antenna Systems. International Engineering Consortium. http://read.pudn.com/downloads157/doc/comm/699605/smart antenna\%20system s.pdf

[26] Fall, K. and Varadhan, K. (2008) The ns Manual (formerly ns Notes and Documentation). The VINT Project.

[27] (2007) The Enhanced Network Simulator (TENS). http://ns-3.blogspot.com/2007/03/enhanced-network-simulator-tens.html

[28] http://cbg.me/2009/02/adding-directional-antenna-and-multiple-interface-supportto-ns-233/

[29] (2014) Support Multiple Interfaces and Multiple Channels. http://itantenna.blogspot.com.au/2012/01/add-multiple-interfaces-and-multiple.ht $\underline{\mathrm{ml}}$ 